\title{
Which methodology is more appropriate to solve eco-driving optimal control problem for conventional vehicles?
}

\author{
D. Maamria ${ }^{a}$, K. Gillet ${ }^{a}$, G. Colin ${ }^{a}$, Y. Chamaillard ${ }^{a}$ and C. Nouillant ${ }^{b}$
}

\begin{abstract}
In this paper, two simplified methods based on Dynamic Programming (DP) to solve an Eco-driving problem for a conventional vehicle equipped with an internal combustion engine are studied. The first method is based on the transformation of a time-based Optimal Control Problem (OCP) into a distance-based OCP while the second is based on solving the time-based OCP directly. The Pontryagin Minimum Principle (PMP) is used to decrease the complexity of the OCP formulation. Based on simulations, the two methods are compared in terms of optimality (fuel consumption) and the time needed to run the DP. The impact of the mesh choice on the optimality of the solution is also investigated.
\end{abstract}

\section{INTRODUCTION}

The announced depletion of fossil fuel sources, climate change due to pollution and an increase in overall energy demands are major challenges for the automotive industry. Generally speaking, energy efficiency is increasingly becoming a major concern and a subject of attention from major international organizations around the world.

Besides the development of alternative fuel sources, the main research directions towards improving energy efficiency in the automotive field focus on fuel efficiency, with a particular emphasis on decreasing carbon dioxide $\left(\mathrm{CO}_{2}\right)$ emissions [1], [2]. Driver support systems [3]-[6] are among the proposed solutions. The idea is the following: there are different ways of driving during a specific journey and they are not equivalent from an energy consumption viewpoint. A driver support system calculates and suggests the speed and the gear-box ratio set points to the driver through an interface integrated in the dashboard of the vehicle with the aim of minimizing the fuel consumption (and/or pollutant emissions) over a given time horizon with various constraints (stops, distance, speed limitations, etc.) [6]-[10].

The design of a driver support system can be formulated as an Optimal Control Problem (OCP) [4], [11]. Usually, the fuel consumption, engine emissions or any combination of both over a fixed time window is the cost function to be minimized [2], [7]. Two dynamics are considered: the speed and the position of the vehicle while the main constraints bear on speed limitations, vehicle stops and traveled distance [12], [13]. This problem was addressed for conventional vehicles in [12], for electric cars in [3], [4], [12] and for hybrid electric cars in [12], [14]-[16].

${ }^{a}$ D. Maamria, K. Gillet, G. Colin and Y. Chamaillard are with Laboratoire PRISME, Université d'Orléans 45072 Orléans, France djamaleddine.maamria@gmail.com

${ }^{b} \mathrm{C}$. Nouillant is with PSA Peugeot Citroën, Direction Recherche Innovation \& Technologies Avancées (DRIA), France
To solve this kind of OCP for conventional vehicles, a three dimensional Dynamic Programming (DP) approach was initially used [2], [17]. In order to reduce the computational time, a two-dimensional approach was subsequently chosen, as suggested in [11], [12], [17]. This twodimensional approach transforms a time-based OCP into a position-based OCP and introduces a terminal tunable cost to penalize the driving cycle duration [11]-[13].

In this paper, an alternative two-dimensional DP approach is investigated for conventional cars. It is based on solving a time-based OCP. A terminal tunable cost is introduced to penalize the total traveled distance. This approach will be compared to the previous one usually used in the literature [11], [12] in terms of optimality (fuel consumption) and computation time (complexity).

The paper is organized as follows. In Section 2, the vehicle model is described. The calculation of eco-driving cycles using DP with two simplified approaches is presented in Section 3. Section 4 discusses numerical results and comparisons for two driving cycles. In light of the results, we draw a conclusion on the appropriate approach to deal with the problem under consideration.

\section{Vehicle Modeling}

\section{A. Motion equations}

The vehicle is modeled on the longitudinal axis. The motion of the vehicle is the result of the forces applied on its body. According to Newton's law of motion, the vehicle speed $v$ satisfies the following differential equation:

$$
\left(m+m_{r o t}\right) \cdot \frac{d v(t)}{d t}=F_{t}(t)-F_{r}(t),
$$

where $F_{t}$ is the traction force to be provided by the engine, $F_{r}$ is the sum of resistance forces and $m$ is the total vehicle mass. The term $m_{r o t}$ is an equivalent mass of the rotating parts. It accounts for the overall inertia of the wheels $\left(n_{\text {tire }}\right.$. $\left.j_{\text {tire }}\right)$ and for that of the engine $\left(j_{r o t}\right)$ :

$$
m_{\text {rot }}=\frac{n_{\text {tire }} \cdot j_{\text {tire }}+j_{\text {rot }}}{r_{\text {tire }}^{2}}
$$

where $r_{\text {tire }}$ is the wheel radius. The force $F_{r}$ comprises the rolling resistance force, the aerodynamic drag force and a force due to the road grade. Its expression is given by:

$$
F_{r}(t)=c_{0}+c_{1} \cdot v(t)+c_{2} \cdot v(t)^{2},
$$

where $c_{i}, i=\{0,1,2\}$ are the coefficients of the road load equation (this expression was employed in [18], [19]). This model considers only the forces in the longitudinal direction. 
Variations of friction parameters during curves, wind forces, and other disturbances are neglected.

\section{B. Transmission}

The driver's torque demand and the vehicle speed are directly calculated from the wheel speed profile, elevation profiles and the gear-box ratio. The resulting torque value $T_{w h}$ can be positive (traction) or negative (braking). The engine torque $T_{\text {eng }}$ is related to the torque required at the wheel $T_{w h}$ by:

$$
T_{w h}(t)=r_{\text {tire }} \cdot F_{t}(t)=\eta_{g b} \cdot R_{g b}(t) \cdot R_{t} \cdot T_{e n g}(t),
$$

where $R_{g b}$ is the gear-box ratio, $\eta_{g b}$ is the gear-box efficiency and $R_{t}$ is the differential ratio. Similarly, the rotational speed $\omega_{e n g}$ of the ICE is related to the vehicle speed $v$ by:

$$
\omega_{\text {eng }}(t)=R_{g b}(t) \cdot R_{t} \cdot \frac{v(t)}{r_{\text {tire }}} .
$$

\section{Internal Combustion Engine (ICE)}

The ICE used here is a diesel engine. The fuel consumption $\dot{m}_{f}(\mathrm{~g} / \mathrm{s})$ is computed through a look-up table (quasistatic map) as a function of the engine rotational speed $\left(\omega_{\text {eng }}\right)$ and the effective engine torque $\left(T_{\text {eng }}\right)$ (see Figure 1):

$$
\dot{m}_{f}=\dot{m}_{f}\left(\omega_{e n g}, T_{e n g}\right) .
$$

The model parameters are summarized in Table I.

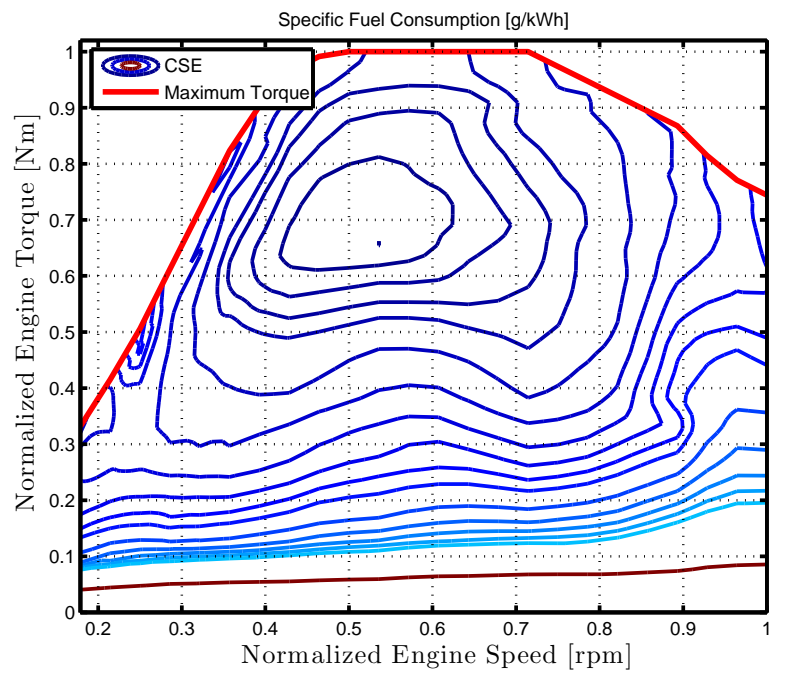

Fig. 1. Specific fuel consumption $(\mathrm{g} / \mathrm{kWh})$ of the internal combustion engine as a function of engine rotational speed and engine torque. For confidentiality reasons, the data are normalized.

\section{ECO-DRIVING}

The so-called eco-driving methodology consists of finding the optimal way to reduce the overall energy consumption [3], [7]. For a fixed road, the objective is to find the best speed profile minimizing the vehicle fuel or power consumption knowing that the vehicle starts from a point $A$ at rest and must reach a destination point $B$ at time $t_{f}$, with a zero velocity. This kind of question can be formulated as an OCP [4], [7].
TABLE I

VEHICLE MODEL PARAMETERS

\begin{tabular}{|llc|}
\hline Acronym & Description & Unit \\
\hline$m$ & Vehicle mass & $\mathrm{kg}$ \\
$r_{\text {tire }}$ & Wheel radius & $\mathrm{m}$ \\
$n_{\text {tire }}$ & Wheel number & - \\
$j_{\text {tire }}$ & Wheel inertia & $\mathrm{kg} \cdot \mathrm{m}^{2}$ \\
$c_{0}$ & Constant coefficient of the road load & $\mathrm{N}$ \\
$c_{1}$ & Linear coefficient of the road load & $\mathrm{N} /(\mathrm{m} / \mathrm{s})$ \\
$c_{2}$ & Quadratic coefficient of the road load & $\mathrm{N} /(\mathrm{m} / \mathrm{s})^{2}$ \\
$\eta_{g b}$ & Gear-box efficiency & - \\
$R_{g b}$ & Gearbox ratio & - \\
$R_{t}$ & Differential ratio & - \\
$\omega_{i d l e}$ & Engine idle speed & $\mathrm{rpm}$ \\
\hline
\end{tabular}

\section{A. OCP formulation}

The cost function (4) to be minimized is the fuel consumption over a fixed time window of duration $t_{f}$.

$$
J=\int_{0}^{t_{f}} \dot{m}_{f}\left(\omega_{e n g}(t), T_{e n g}(t)\right) d t .
$$

The control variable $u$ is composed of two components: the engine torque $T_{e n g}$ and the gear-box ratio $R_{g b}$ :

$$
u(t)=\left[T_{e n g}(t), R_{g b}(t)\right] .
$$

This optimization is carried out under the following dynamical constraints:

$$
\begin{aligned}
\frac{d v(t)}{d t} & =f(v(t), u(t)), v(0)=0 \\
\frac{d x(t)}{d t} & =v(t), x(0)=0
\end{aligned}
$$

where $x$ is the position of the vehicle and the function $f$ is calculated by combining $(1,2,3)$ :

$f=\frac{1}{m+m_{\text {rot }}}\left(-c_{0}-c_{1} \cdot v-c_{2} \cdot v^{2}+\frac{\eta_{g b}}{r_{\text {tire }}} \cdot R_{g b} \cdot R_{t} \cdot T_{\text {eng }}\right)$.

Since the speed, the engine torque and the gear-box ratio are limited and the final position and the final speed are fixed, the optimization must be performed under the following state and input constraints:

$$
\begin{aligned}
v(t) & \in\left[0, v_{\max }(x(t))\right], \\
T_{\text {eng }}(t) & \in\left[T_{\min }\left(\omega_{\text {eng }}(t)\right), T_{\max }\left(\omega_{\text {eng }}(t)\right)\right], \\
x\left(t_{f}\right) & =D \\
v\left(t_{f}\right) & =0,
\end{aligned}
$$

where $D$ is the total traveled distance, $T_{\min }$ and $T_{\max }$ are given by look-up tables as a function of the engine rotation speed $\omega_{\text {eng }}$. The speed limitations are given as a function of the vehicle position [2], [3] and not of time, as shown in Figure 2 (the blue line is the initial driving cycle and the dashed line represents the chosen speed limits).

\section{B. Dynamic Programming (DP)}

In DP, the calculation of the optimal trajectories is based on the Bellman principle while searching from the final state backward in time. The Bellman principle states that an optimal policy has the property that whatever the initial 


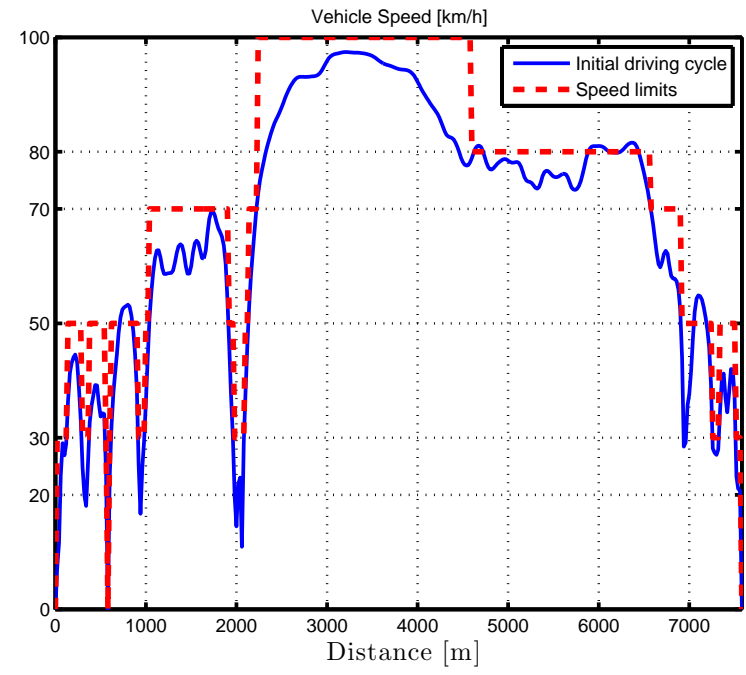

Fig. 2. Speed Limits $(\mathrm{km} / \mathrm{h})$ as a function of the vehicle position (m)

state and initial decision are, the remaining decisions must constitute an optimal policy with regard to the state resulting from the first decision [20], [21].

Considering the cost function $J$ to be minimized:

$$
J=\int_{0}^{t_{f}} L(X(t), u(t), t) d t
$$

from a mathematical viewpoint, Bellman's principle can be formulated as follows: let $t \in\left[0, t_{f}\left[\right.\right.$ and $\mathbf{X}(t) \in \mathbb{R}^{n}$ be given, then for all real $r \in\left[t, t_{f}\right]$, the cost-to-go function $V$ satisfies:

$V(X(t), t)=\min _{u \in \mathcal{U}}\left\{\int_{t}^{r} L(X(\tau), u(\tau), \tau) d \tau+V(X(r), r)\right\}$

This equation is solved recursively. The problem is meshed: $\Delta t$ for time $t_{k}, \Delta \mathbf{X}$ for the state variables $\mathbf{X}\left(t_{k}\right)=\mathbf{X}_{k}$ and $\Delta u$ for the inputs $\mathbf{u}_{k}$. Then, proceeding backward from the final state at $t=t_{f}$, the algorithm computes the cost from one state to another until the initial time $t=0$. Finally, the optimal control trajectories are simulated in order to obtain the optimal state trajectories. However, problems of computation time and of memory allocation generally prevent the method from computing optimal solutions of problems with a long time horizon and a state dimension greater than three. This is the known as the "curse of dimensionality".

\section{Speed trajectory computation}

To compute an eco-driving cycle, the following constraints [7] have to be included:

- the same final distance $x\left(t_{f}\right)$, the same number of stops and the same duration $t_{f}$ as the initial driving cycle,

- the vehicle speed limitations depending on the position of the vehicle $(x)$.

Then, the objective is to find a new driving cycle that takes these constraints into account and leads to a lower fuel consumption. In the case of eco-driving cycles, vehicle stops are relevant in distance and not in time [12].
To calculate the optimal velocity profile, two simplified approaches are investigated. The Pontryagin Minimum Principle (PMP) [22] is used to decrease the complexity of the OCP. After, the obtained simplified OCPs are solved using DP.

1) Time method: In this approach, the OCP is solved in the time domain. The associated Hamiltonian $H_{1}$ is defined by:

$$
H_{1}(v, u, \lambda, \mu)=\dot{m}_{f}(v, u)+\lambda \cdot f(v, u)+\mu \cdot v,
$$

where $\lambda$ and $\mu$ are the adjoint variables associated to $v$ and $x$, respectively. From the first order optimality conditions, the adjoint states $\lambda$ and $\mu$ are defined by:

$$
\begin{aligned}
\dot{\lambda} & =-\frac{\partial H_{1}}{\partial v}, \\
\dot{\mu} & =-\frac{\partial H_{1}}{\partial x}=0 .
\end{aligned}
$$

From the second equation, $\mu$ is constant and its value is calculated to satisfy the final constraint on $x: x\left(t_{f}\right)=D$. So, for a fixed value of $\mu$ one can define the following simplified OCP:

$$
\left(O C P_{1}\right): \min _{u} \int_{0}^{t_{f}}\left[\dot{m}_{f}(v, u)+\mu \cdot v\right] d t
$$

under the dynamics of $v$ in (5), the final constraint in (10) and the constraints $(7,8)$. The final constraint (9) on the vehicle position is satisfied by finding the value of the constant tunable parameter $\mu$. In this method, the final time $t_{f}$ and the time step are fixed.

2) Space method: In this approach, the OCP is solved in the space domain. From the dynamics of $x$, we can write, when $v$ is not zero:

$$
d t=\frac{d x}{v} .
$$

This expression is used to transform a time-based OCP into a position-based OCP as follows:

1) The cost function to be minimized becomes of the form:

$$
J_{\text {mod }}=\int_{0}^{D} \frac{\dot{m}_{f}(v, u)}{v} d x .
$$

2) The dynamics of $t$ and $v$ are of the form:

$$
\begin{aligned}
& \frac{d v}{d x}=\frac{f(v, u)}{v} \\
& \frac{d t}{d x}=\frac{1}{v} .
\end{aligned}
$$

3) The final constraints: $t(D)=t_{f}, v(D)=0$.

The Hamiltonian $\mathrm{H}_{2}$ associated with this new OCP is defined by:

$$
H_{2}\left(v, u, p_{1}, p_{2}\right)=\frac{\dot{m}_{f}(v, u)}{v}+p_{1} \cdot \frac{f(v, u)}{v}+p_{2} \cdot \frac{1}{v},
$$

where $p_{1}$ and $p_{2}$ are the adjoint variables associated to $v$ and $t$, respectively. The adjoint states $p_{1}$ and $p_{2}$ are given by:

$$
\begin{aligned}
& \dot{p}_{1}=-\frac{\partial H_{2}}{\partial v}, \\
& \dot{p}_{2}=-\frac{\partial H_{2}}{\partial t}=0 .
\end{aligned}
$$


From the second equation, $p_{2}$ is constant and its value is calculated to satisfy the final constraint on $t: t(D)=t_{f}$. So, for a fixed value of $p_{2}$, we can define the following simplified OCP:

$$
\left(O C P_{2}\right): \min _{u} \int_{0}^{D}\left[\dot{m}_{f}(v, u)+p_{2}\right] \frac{d x}{v}
$$

under the dynamics of $v$ in (12), the final constraint $v(D)=$ 0 and the constraints $(7,8)$. The constraint on the final time $t(D)=t_{f}$ is satisfied by finding the value of the constant tunable parameter $p_{2}$. This method is similar to the one used in [11], [12] where an additional tunable term was added to the cost function as a terminal cost $\beta \cdot t_{f}$. The constant tunable parameter $\beta$ penalizes the final time to obtain almost the same time duration as the initial driving cycle. In this method, final position $x\left(t_{f}\right)$ and distance step are fixed.

\section{NUMERICAL RESULTS AND COMPARISON}

The simulation results are obtained for a conventional vehicle (1930 kg curb weight) equipped with a diesel engine. Figure 1 describes the specific fuel consumption of the engine as a function of the normalized engine torque and the normalized engine rotational speed.

This Section is divided into two parts. In the first part, the two methods (time and space) are compared. A short driving cycle of duration $360 \mathrm{~s}$ and traveled distance of $6.9 \mathrm{~km}$ with only one speed limit of $80 \mathrm{~km} / \mathrm{h}$ is considered. The objective is to analyze the solutions of $\left(\mathrm{OCP}_{1}\right)$ and $\left(\mathrm{OCP}_{2}\right)$ in terms of optimality (fuel consumption) and computation time. Based on the conclusion drawn from this analysis, we decide which methodology is the most appropriate to solve the OCP defined in Section III-A.

In the second part, the space method is used. The impact of the mesh choice on the optimality of the solution and the state trajectories is studied: a more realistic driving cycle extracted from the Worldwide harmonized Light vehicles Test Cycle (WLTC), namely a cycle of duration 588 s and traveled distance of $7.6 \mathrm{~km}$ is used. The speed limits presented in Figure 2 are considered.

\section{A. Comparison of the time and the space methods for a} (short) driving cycle

The $\left(\mathrm{OCP}_{1}\right)$ and $\left(\mathrm{OCP}_{2}\right)$ are solved for various values of the constant parameters $\mu$ and $p_{2}$. The following mesh was chosen: a spacing of $d u=1 \mathrm{~N} . \mathrm{m}$ for the engine torque $T_{\text {eng }}$, of 1 for the gear-box ratio $R_{g b}$ (from 1 to 6 ) and of $d v=$ $0.01 \mathrm{~m} / \mathrm{s}$ for the vehicle speed. For $\left(\mathrm{OCP}_{1}\right)$, the time step is $d t=1 \mathrm{~s}$ and for $\left(\mathrm{OCP}_{2}\right)$, the distance step is $d x=20 \mathrm{~m}$. The results obtained in terms of fuel consumption, traveled distance and final time are given in Figure 3 (respectively in Figure 4). Each point in this figure is obtained for a fixed value of $\mu$ (respectively of $p_{2}$ ).

These results show that the relationships between the fuel consumption, the traveled distance and the final time are monotonic: the fuel consumption increases when the distance increases (see Figure 3) while it decreases when the final time increases (see Figure 4).

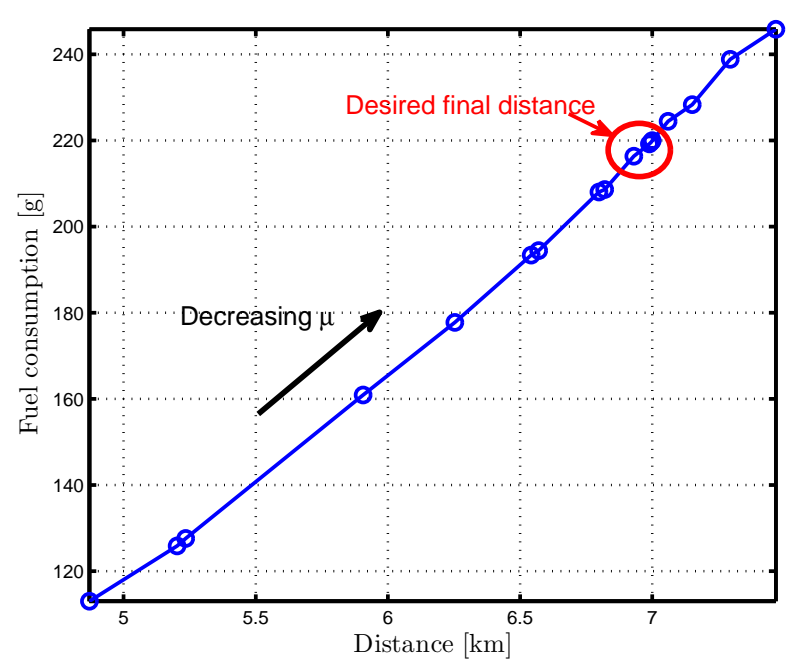

Fig. 3. Fuel consumption [g] as a function of the traveled distance [km]. Each point in this figure is obtained for a fixed value of $\mu$ in $\left(\mathrm{OCP}_{1}\right)$.

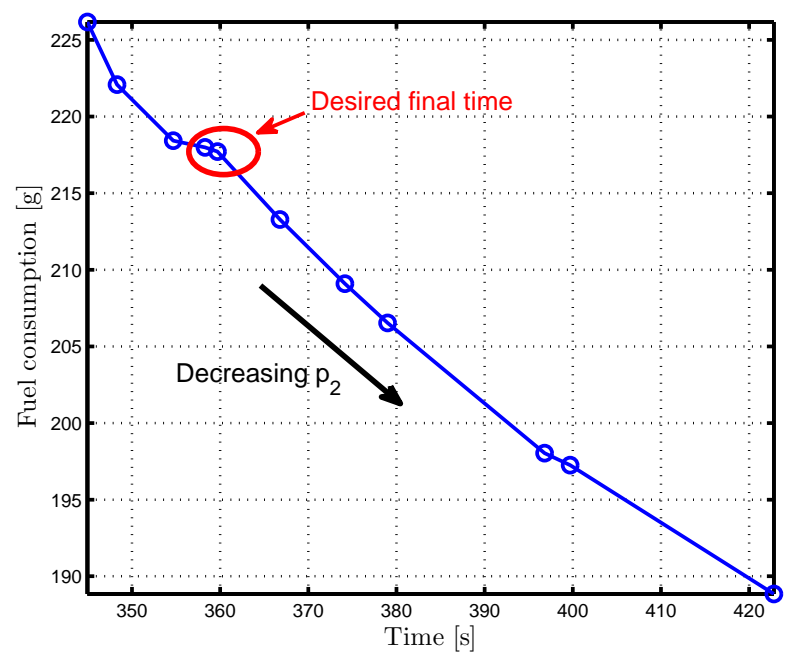

Fig. 4. Fuel consumption [g] as a function of the final time $t_{f}[\mathrm{~s}]$. Each point in this figure is obtained for a fixed value of $p_{2}$ in $\left(\mathrm{OCP}_{2}\right)$.

To make a fair comparison between the two methods, the final time $t_{f}$ and the traveled distance $x\left(t_{f}\right)$ have to be almost the same (surrounded regions with red circles in Figures 3 and 4). The values of $\mu$ and $p_{2}$ ensuring these two conditions can be iteratively determined (for example by using a dichotomy or a Newton method). The vehicle speed trajectories satisfying these two conditions for the two methods are given in Figure 5: the two speed trajectories are comparatively close. The fuel consumption and the time needed to run the DP for each OCP are the following:

- The time method: the fuel consumption is $216.4 \mathrm{~g}$ and the time needed to solve the $\left(\mathrm{OCP}_{1}\right)$ is $1200 \mathrm{~s}$.

- The space method: the fuel consumption is $217.8 \mathrm{~g}$ and the time needed to solve the $\left(\mathrm{OCP}_{2}\right)$ is $1150 \mathrm{~s}$.

The two approaches are very close in terms of fuel consumption (the time method is better than the space method) and 


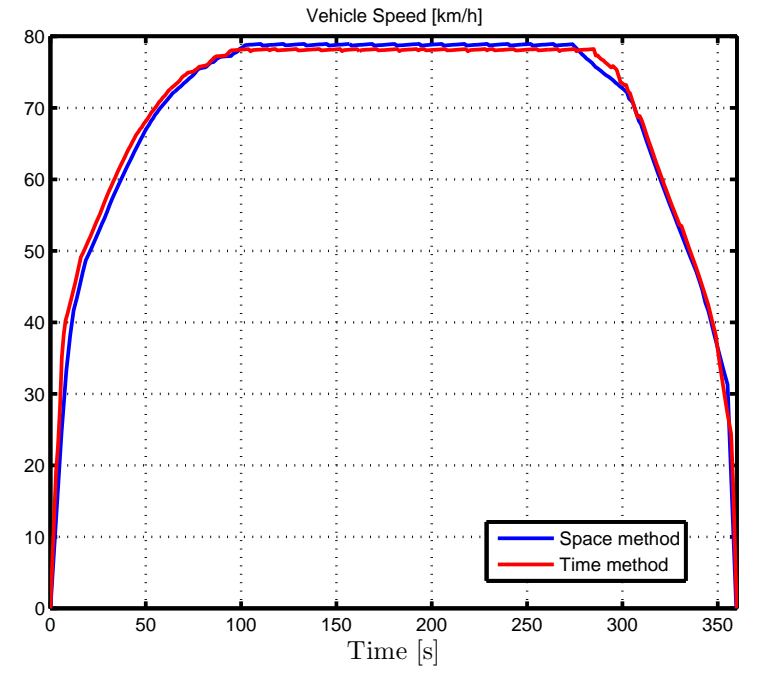

Fig. 5. Vehicle speed $[\mathrm{km} / \mathrm{h}]$ as a function of time $[\mathrm{s}]$

computation time (the space method is faster than the time method).

The distance and time steps for the two approaches are presented in Figures 6 and 7, respectively. These figures show that the time method is more accurate at low vehicle speed (as it has the lowest $\delta x$ and $\delta t$ at low vehicle speed) while the space method is more accurate at high vehicle speed (as it has the lowest $\delta x$ and $\delta t$ at high vehicle speed).

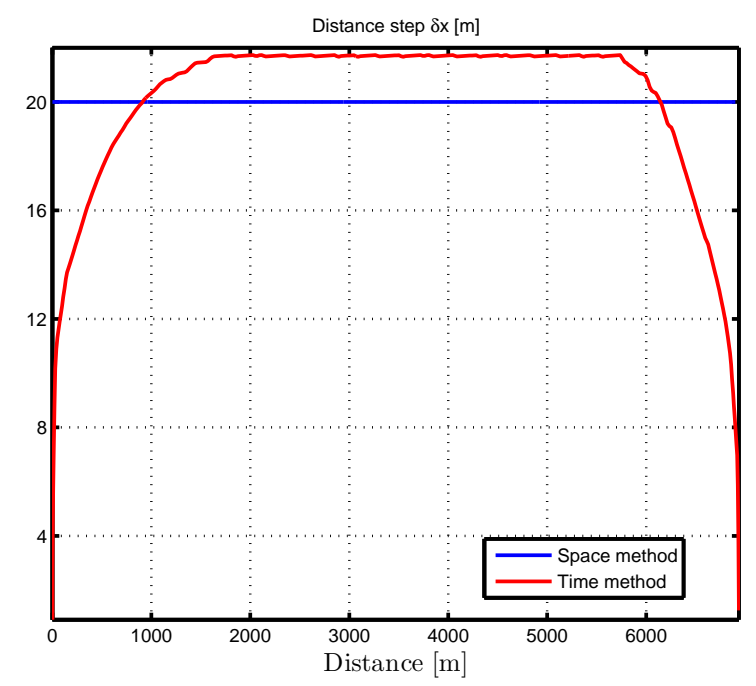

Fig. 6. Distance step $\delta x[\mathrm{~m}]$

As mentioned before, the speed limits are a function of the vehicle position. The drawback of the time method is the difficulty of of taking this dependance into account: the calculation time will increase (the speed limits considered for the short driving cycle are constant and independent of the vehicle position, and the calculation time for the time method is $4.2 \%$ higher than with the space method). On the other hand, the induced sub-optimality on the fuel consumption is small (less than $0.6 \%$ ). For these reasons, the space method is used in the next section for the ecodriving cycle computation.

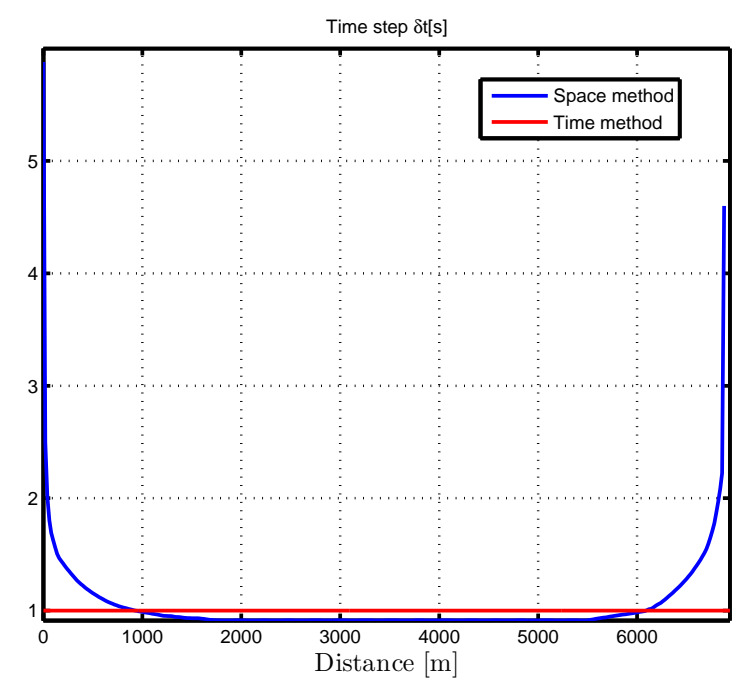

Fig. 7. Time step $\delta t[\mathrm{~s}]$

\section{B. Sensitivity study of the space method to the mesh choice}

The space method is used to calculate optimal speed trajectories with the speed limits presented in Figure 2. Several meshes are tested in order to find a trade-off between the optimality of the solution and the time needed to solve the OCP (14) by the DP. The obtained speed trajectories are shown in Figure 8 versus distance and in Figure 9 versus time. The fuel consumption [L/100 km] and the computation time $\alpha$ [s] needed to run the DP are given in Table II.

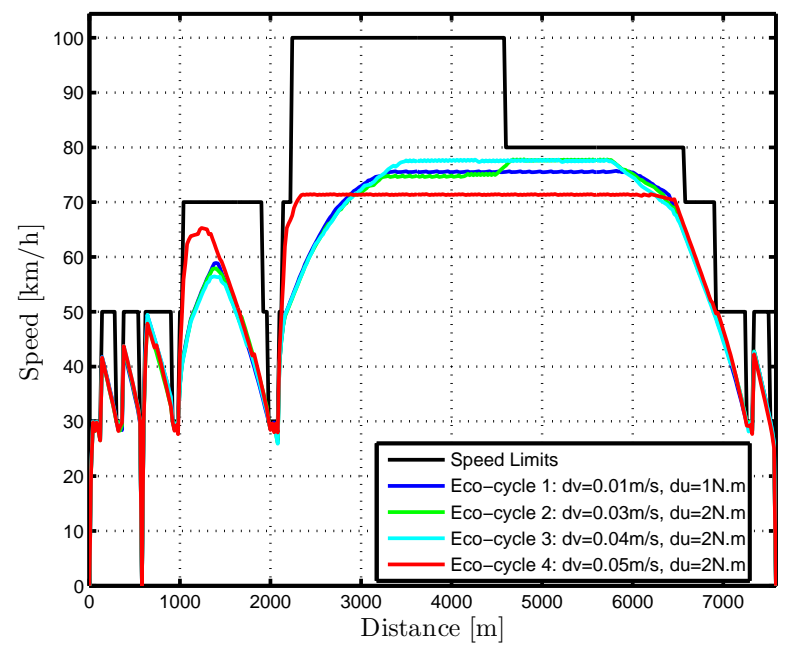

Fig. 8. Vehicle speed $[\mathrm{km} / \mathrm{h}]$ vs distance $[\mathrm{m}]$.

Figures 8 and 9 show that the speed trajectories are impacted by the mesh choice. For the meshes $[d v=0.03 \mathrm{~m} / \mathrm{s}$, $d u=2 \mathrm{~N} . \mathrm{m}]$ and $[d v=0.04 \mathrm{~m} / \mathrm{s}, d u=2 \mathrm{~N} . \mathrm{m}]$, the speed trajectories are relatively close to the trajectory calculated for $[d v=0.01 \mathrm{~m} / \mathrm{s}, d u=1 \mathrm{~N} . \mathrm{m}]$ (which is considered as 
TABLE II

TIME $\alpha$ NEEDED TO RUN THE DP AND FUEL CONSUMPTION.

\begin{tabular}{|l|c|c|}
\hline & Consumption [L/100 km] & $\alpha[\mathrm{s}]$ \\
\hline Initial Cycle & 5.60 & - \\
\hline Eco-cycle 1: $d v=0.01, d u=1$ & 3.72 & 1620 \\
\hline Eco-cycle 2: $d v=0.03, d u=2$ & 3.76 & 240 \\
\hline Eco-cycle 3: $d v=0.04, d u=2$ & 3.76 & 120 \\
\hline Eco-cycle 4: $d v=0.05, d u=2$ & 3.83 & 77 \\
\hline
\end{tabular}

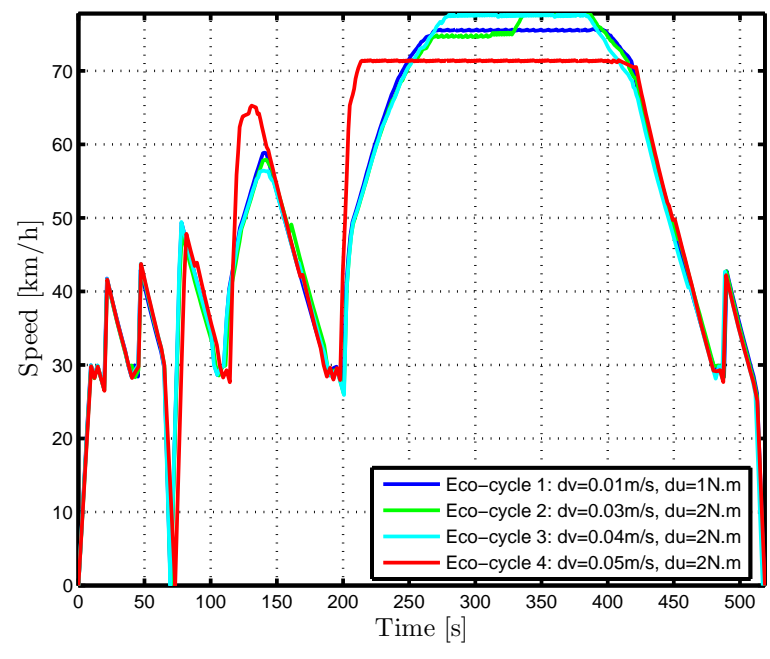

Fig. 9. Vehicle speed $[\mathrm{km} / \mathrm{h}]$ vs Time $[\mathrm{s}]$.

a reference in this study). The Eco-cycle 4 calculated for $[d v=0.05 \mathrm{~m} / \mathrm{s}, d u=2 \mathrm{~N} . \mathrm{m}]$ is quite different from Ecocycle 1 , mainly at high vehicle speeds.

To find a trade-off between the optimality of the different solutions and the computation time, the fuel consumptions given in Table II are compared. For the Eco-cycle 3, the fuel consumption is very close to the fuel consumption for Eco-cycle 1 (with an induced sub-optimality less than $1 \%$ ) while the computation time is divided by 13 . For the Eco-cycle 4, the induced sub-optimality compared to Eco-cycle 1 is $3 \%$ while the time needed to run the DP is divided by 21. Thus, the DP solution for the mesh $[d v=0.04 \mathrm{~m} / \mathrm{s}, d u=2 \mathrm{~N} . \mathrm{m}]$ can be considered as accurate enough to guarantee a quasi-optimal fuel consumption while requiring an acceptable computation time. A similar analysis was conducted for other normalized driving cycles (NEDC, WLTC, Urban Artemis, Artemis Rural and Artemis highway cycles) and the conclusion reached is that it is possible to find a trade-off between the optimality of the solution and the computation time of the DP.

\section{CONCLUSION}

The eco-driving problem for conventional vehicles has been addressed. This problem is formulated as an OCP aiming at minimizing fuel consumption. Two simplified approaches (time and space approaches) have been investigated to solve this optimization problem. The two approaches are very close in terms of fuel consumption and computation time. On the other hand, the space method is more appro- priate for taking speed limits into account (as they are given as a function of the vehicle position).

Additionally, the impact of the mesh choice has been studied. Based on the numerical results presented here, it is possible to find a trade-off between the optimality of the solution (fuel consumption) and the computation time of the DP.

\section{REFERENCES}

[1] S.-Y. Kim, D.-J. Shin, and H.-J. e. a. Yoon, "Development of ecodriving guide system," In SAE asia pacific automotive engineering conference, pp. 1636-1641, 2011.

[2] F. Mensing, R. Trigui, and E. Bideaux, "Vehicle trajectory optimization for application in eco-driving," IEEE Vehicle Power and Propulsion Conference, pp. 1-6, 2011.

[3] W. Dib, A. Chasse, P. Moulin, A. Sciarretta, and G. Corde, "Optimal energy management for an electric vehicle in eco-driving applications,' Control Engineering Practice, vol. 29, pp. 299-307, 2014.

[4] N. Petit and A. Sciarretta, "Optimal drive of electric vehicles using an inversion-based trajectory generation approach," 18th IFAC World Congress, vol. 18, pp. 14519-14526, 2011.

[5] T. van Keulen, B. de Jager, D. Foster, and M. Steinbuch, "Velocity trajectory optimization in hybrid electric trucks," in Proc. American Control Conference, pp. 5074-5079, 2010.

[6] L. Eriksson and L. Nielsen, Modeling and Control of Engines and Drivelines. Wiley, 2014.

[7] F. Mensing, E. Bideaux, R. Trigui, J. Ribet, and B. Jeanneret, "Ecodriving: an economic or ecologic driving style?," Transportation Research Part C: Emerging Technologies, vol. 38, pp. 110-121, 2014.

[8] M. Miyatake, M. Kuriyama, and Y. Takeda, "Theoretical study on ecodriving technique for an electric vehicle considering traffic signals," in Proc. 9th IEEE Int. Conf. Power Electronics Drive Systems, pp. 733 738, 2011.

[9] E. Ozatay, U. Ozguner, J. Michelini, and D. Filev, "Analytical solution to the minimum energy consumption based velocity profile optimization problem with variable road grade ?", 19th World Congress, pp. 7541-754, 2014.

[10] M. A. S. Kamal, M. Mukai, J. Murata, and T. Kawabe, "On board eco-driving system for varying road-traffic environments using model predictive control," IEEE International Conference on Control Applications, pp. 1636-1641, 2010.

[11] V. Monastyrsky and I. Golownykh, "Rapid computation of optimal control for vehicles," Transportation Research Part B, vol. 27, pp. 219227, 1993.

[12] F. Mensing, Optimal energy utilization in conventional, electric and hybrid vehicles and its application to eco-driving. $\mathrm{PhD}$ thesis, INSA Lyon, 2013.

[13] A. Sciarretta, G. D. Nunzio, and L. L. Ojeda, "Optimal ecodriving control: Energy-efficient driving of road vehicles as an optimal control problem," IEEE Control Systems Magazine, vol. 35.5, pp. 71-90, 2015.

[14] H. Bouvier, G. Colin, and Y. Chamaillard, "Determination and comparison of optimal eco-driving cycles for hybrid electric vehicles," European Control Conference, pp. 142-147, 2015.

[15] T. S. Kim, C. Manzie, and R. Sharma, "Two-stage optimal control of a parallel hybrid vehicle with traffic preview," IFAC Proceedings Volumes, vol. 44, p. 21152120, 2011.

[16] E. Hellstrm, J. slund, and L. Nielsen, "Design of an efficient algorithm for fuel-optimal look-ahead control," Control Engineering Practice, vol. 18, p. 13181327, 2010.

[17] J. Hooker, "Optimal driving for single-vehicle fuel economy," Transportation Research-A, pp. 183-201, 1988.

[18] L. Guzzella and A. Sciarretta, Vehicle propulsion systems. Springer, 2013.

[19] S-B. Ebbesen, Optimal sizing and control of hybrid electric vehicles. $\mathrm{PhD}$ thesis, ETH Zurich, 2012.

[20] D. Bertsekas, Dynamic programming and optimal control. Athena Scientific, 2012.

[21] A.-E. Bryson and Y.-C. Ho, Applied optimal control. Ginn and Company: Waltham, MA, 1969.

[22] L.-S. Pontryagin, V.-G. Boltyanskii, R.-V. Gamkrelidze, and E.-F. Mishchenko, The mathematical theory of optimal processes. Interscience Publishers John Wiley \& Sons, Inc. New York, London, 1962. 